\title{
Structure-function profile of MmpL3, the essential mycolic acid transporter from Mycobacterium tuberculosis
}

Juan Manuel Belardinelli, Amira Yazidi, Liang Yang, Lucien Fabre, Wei Li, Benoit Jacques, Shiva kumar Angala, Isabelle Rouiller, Helen I. Zgurskaya*, Jurgen Sygusch*, and Mary Jackson*

*E-mails: Mary.Jackson@colostate.edu; elenaz@ou.edu; jurgen.sygusch@umontreal.ca

\section{Supporting Information}

$\underline{\text { Table of contents }}$

Supplementary Figures

Figure S1: Purification and characterization of CmpL1.

Figure S2: MmpL3 quaternary structure derived by superposition of the MmpL3 subunits onto the CmpL1 trimer.

Figure S3: Transmembrane topology of MmpL3.

Figure S4: Multiple alignments of MmpL proteins from $\mathrm{Mtb}$ and $\mathrm{Cgl}$.

Figure S5: Effect of thiol-reactive probes on mycolic acid export by $M s m g \Delta m m p L 3$ strains expressing MmpL3 mutants with cysteine substitutions at positions Q40, Y235, P625 and V694.

Supplementary Tables

Table S1: I-TASSER free modeling.

Table S2: Drug susceptibility of Msmg $\Delta m m p L 3$ strains expressing mutated variants of MmpL3.

Supplementary Movie S1: Movie showing the 3D EM negative staining map and the superposition of the CmpL1 structural model refined against the EM map.

Supplementary Methods

Supplementary References 


\section{Figure S1: Purification and characterization of CmpL1 and MmpL3.}

(a) CmpL1, MmpL3 and their high molecular weight detergent complexes were purified from $\mathrm{Cgl}$ cells as described in the Supplementary Methods. Protein samples were treated with the indicated concentrations of the amine-reactive crosslinker, DSP, separated by $8 \%$ SDS-PAGE and visualized by immunoblotting using an anti-Strep antibody. The positions of the expected monomeric (M, MW = $101 \mathrm{kDa}$ for MmpL3; MW = $83 \mathrm{kDa}$ for CmpL1), dimeric (D) and trimeric (T) species are indicated. Elution profiles obtained by size exclusion chromatography of CmpL1 (b) and AcrB (c) as a reference standard and measured at $280 \mathrm{~nm}$. The Superdex 200 GE 3.2/300 column was previously calibrated using proteins of defined molecular weight (Thyroglobulin (bovine) $670 \mathrm{kDa}$, gamma-globulin (bovine) $158 \mathrm{kDa}$, Ovalbumin (chicken) $44 \mathrm{kDa}$, Myoglobin (horse) $17 \mathrm{kDa}$, Vitamin B12 $1.35 \mathrm{kDa}$ ). The estimated molecular weights for the peak fractions are shown in red. The minor high MW fraction for AcrB is situated on the shoulder of the major low MW fraction of AcrB and as result the MW of the minor fraction is underestimated.

a

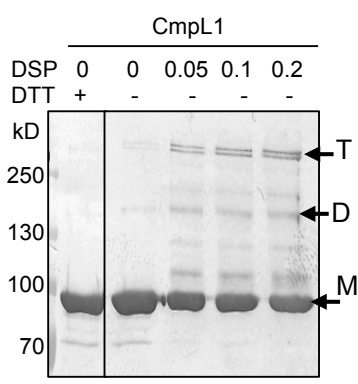

b

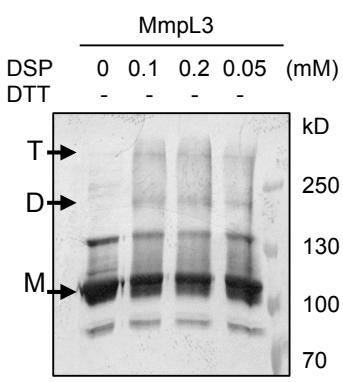

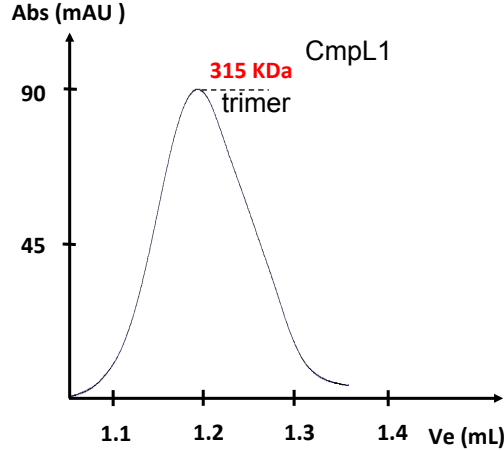

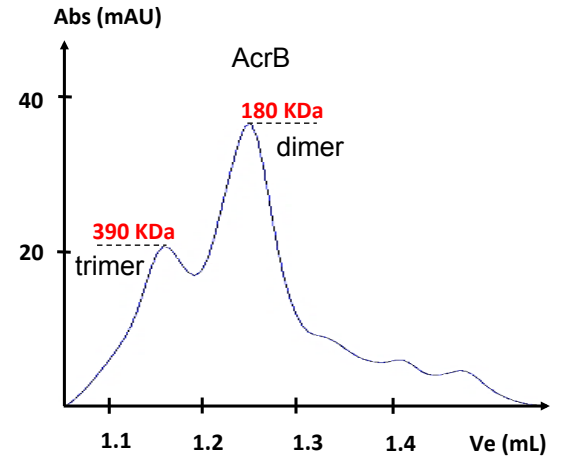


Figure S2: MmpL3 quaternary structure derived by superposition of the MmpL3 subunits onto the CmpL1 trimer. The transmembrane regions of the MmpL3 subunits are colored gray, pale green and pale cyan to facilitate their discernment. The periplasmic domains PN (34-173) and PC (417-551) are colored deep salmon and cyan, respectively. The C-terminal region (729-944) is colored purple.

G543 whose mutation to Cys abolishes transport activity is shown in a loop at the subunit interface, while residue D550 whose mutation to Cys reduces activity is shown at the periplasmic channel entrance. When mutated to Cys, residues S33 and V105 are found inaccessible to the FM probe. The orientation of V105 at the subunit interface makes it solvent inaccessible. Likewise, S33 caps TMS-1 and its location at the outer leaflet of the cytoplasmic membrane most likely makes it inaccessible to the probe.
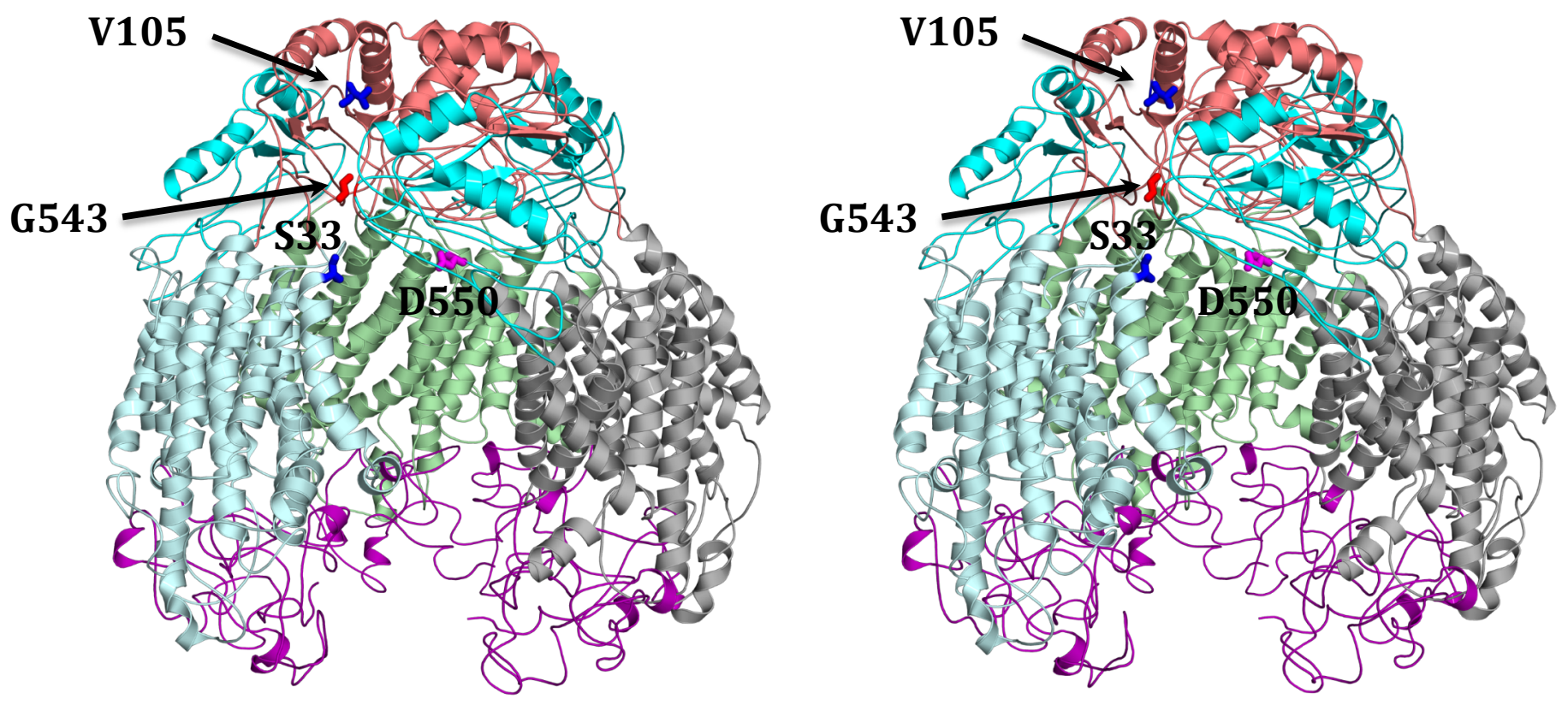


\section{Figure S3: Transmembrane topology of MmpL3}

(a) Expression of the Cys-substituted $\Delta$ Cys-MmpL3 variants in $C g l$. $C g l$ cells producing the $\Delta$ Cys-MmpL3 variants containing Cys substitutions at the indicated positions were induced with IPTG and membrane fractions were isolated as described in the Supplementary Methods. Upon labeling with fluorescein-5-maleimide (FM), reactions were quenched with DTT, membrane proteins solubilized with detergent, and $\Delta$ Cys-MmpL3 variants purified by affinity chromatography. The fractions containing the eluted proteins were separated by $8 \%$ SDS-PAGE, fluorescence quantified using a Storm Imager (see Fig. 4b), and the protein bands were visualized with silver nitrate staining (shown below). M,

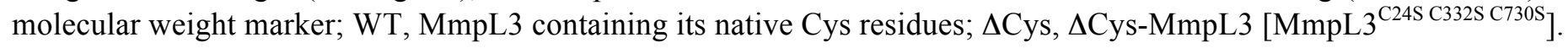

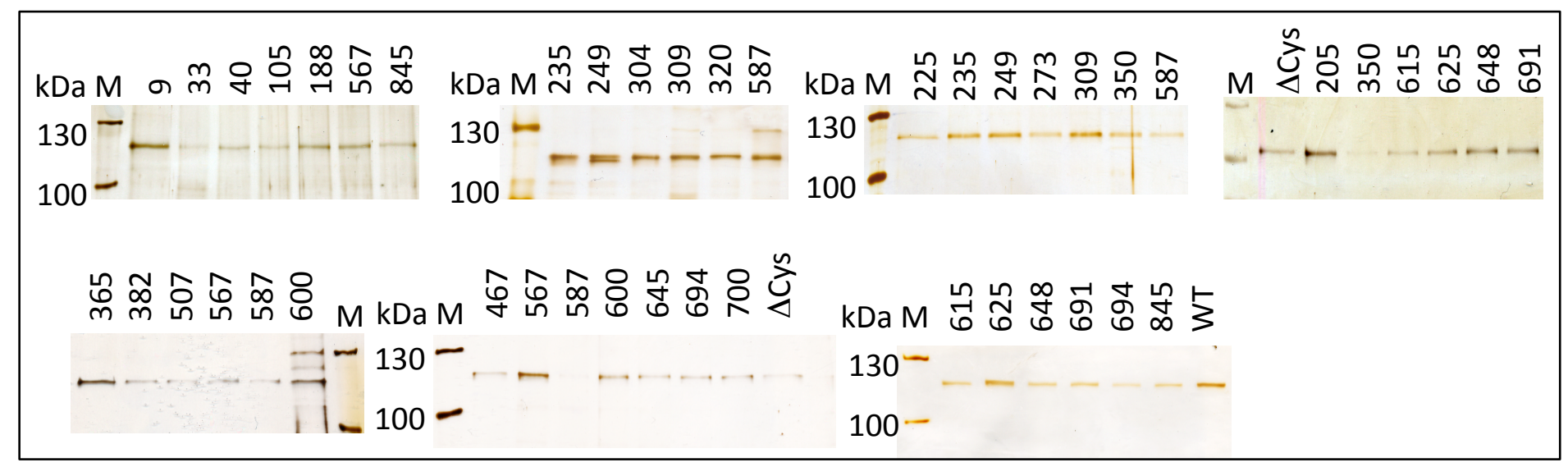

(b) Shown in the table and figure below is the transmembrane topology of MmpL3 from Mtb based on the MmpL3 model generated in this study.

The positions of the Cys-mutated $\Delta$ Cys-MmpL3 variants used in the topology studies are represented as stars. Yellow stars indicate residues to which the fluorescent probe, FM, bound; black stars indicate unlabeled residues.

Squares indicate functional residues. Red squares indicate the position of the mutations that failed to rescue the Msmg $\Delta m m p L 3$ knock-out mutant; green squares indicate the position of mutations that reduced MmpL3 activity. Note that residues Q304, P625 and S691 that are labeled as stars on the figure were identified as functional residues in our study. These residues are further annotated with a black asterisk.

The positions of the three GFP fusions generated in MmpL3 from M. smegmatis are indicated by arrows. The graphical model was generated using TOPO2.

\begin{tabular}{|c|c|c|c|c|c|c|c|c|c|c|c|c|c|c|c|}
\hline Domains & $\begin{array}{c}\text { TMS- } \\
1\end{array}$ & PN & $\begin{array}{c}\text { TMS- } \\
2\end{array}$ & $\begin{array}{c}\text { TMS- } \\
3\end{array}$ & $\begin{array}{c}\text { TMS- } \\
4\end{array}$ & $\begin{array}{c}\text { TMS- } \\
5\end{array}$ & $\begin{array}{c}\text { TMS- } \\
6\end{array}$ & $\begin{array}{c}\text { TMS- } \\
7\end{array}$ & PC & $\begin{array}{c}\text { TMS- } \\
8\end{array}$ & $\begin{array}{c}\text { TMS- } \\
9\end{array}$ & $\begin{array}{c}\text { TMS- } \\
10\end{array}$ & $\begin{array}{c}\text { TMS- } \\
11\end{array}$ & $\begin{array}{c}\text { TMS- } \\
12\end{array}$ & $\begin{array}{c}\mathrm{C}- \\
\text { terminus }\end{array}$ \\
\hline Residues & $14-33$ & $\begin{array}{l}34- \\
173 \\
\end{array}$ & $\begin{array}{l}174- \\
199 \\
\end{array}$ & $\begin{array}{c}208- \\
224 \\
\end{array}$ & $\begin{array}{c}238- \\
264 \\
\end{array}$ & $\begin{array}{c}271- \\
301 \\
\end{array}$ & $\begin{array}{c}306- \\
338 \\
\end{array}$ & $\begin{array}{c}396- \\
415 \\
\end{array}$ & $\begin{array}{l}417- \\
551\end{array}$ & $\begin{array}{c}552- \\
576 \\
\end{array}$ & $\begin{array}{c}583- \\
601 \\
\end{array}$ & $\begin{array}{c}625- \\
648 \\
\end{array}$ & $\begin{array}{c}660- \\
690 \\
\end{array}$ & $\begin{array}{c}697- \\
728 \\
\end{array}$ & $729-944$ \\
\hline
\end{tabular}




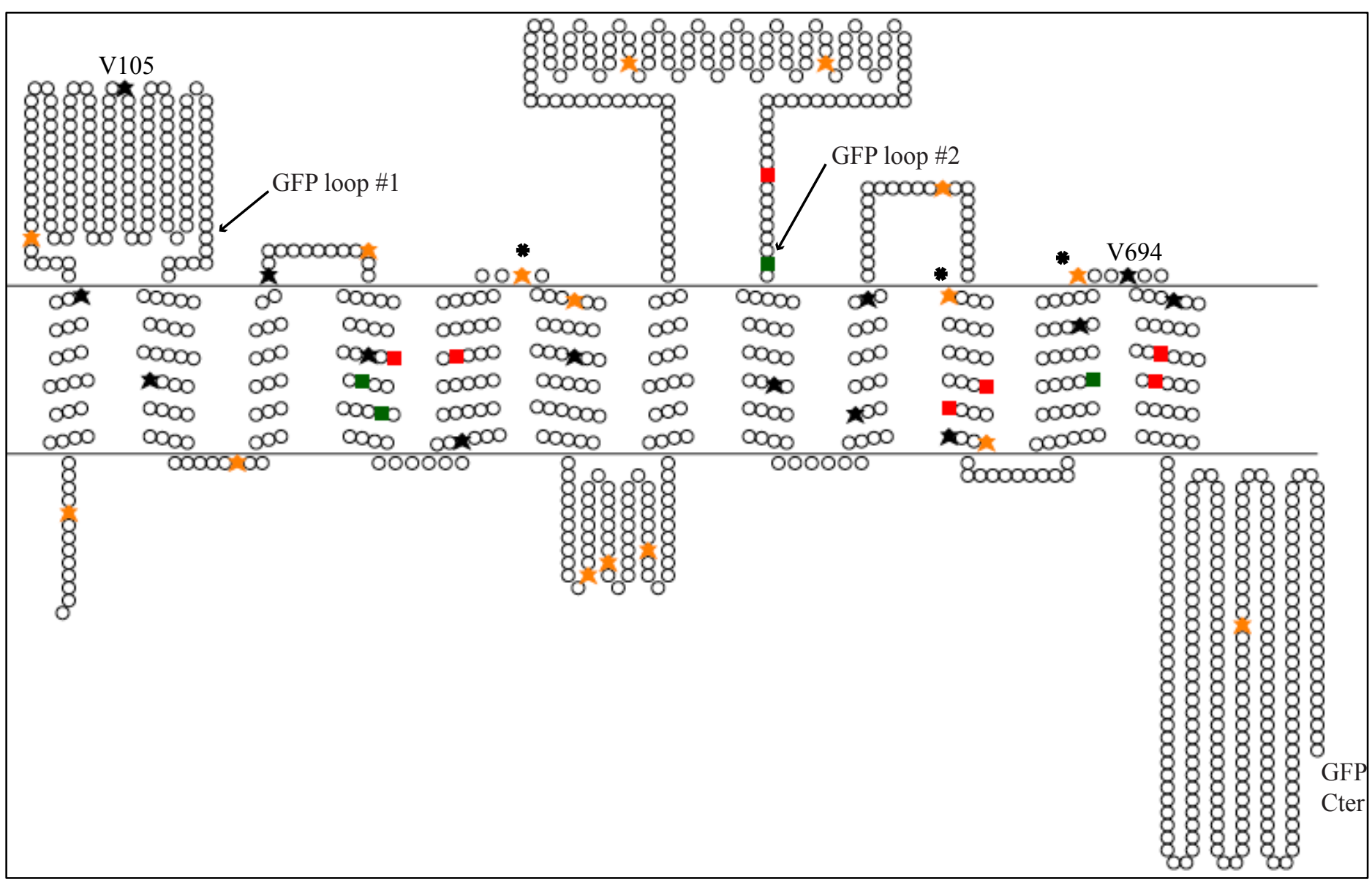


Figure S4: Multiple alignments of MmpL proteins from $\mathbf{M t b}$ and $\boldsymbol{C g l}$.

Alignments using Cluster Omega (version 1.2.1) of two conserved regions of $\mathrm{MmpL} / \mathrm{CmpL}$ family proteins. Alignment (a) maps to the first half of the proteins; alignment (b) maps to the second half. Identical residues are in black, similar residues in gray. Asterisks below the alignments denote essential MmpL3 residues. Arrowheads denote residues whose mutation to Cys significantly reduces the activity of MmpL3.

(a)

MmpL1

MmpL2

MmpL 9

MmpL 5

MmpL 4

MmpL12

MmpL10

MmpL 8

MmpL1 1

MmpL3

CmpL1

MmpL13a

MmpL1

MmpL2

MmpL 9

MmpL 5

MmpL 4

MmpL12

MmpL10

MmpL 8

MmpL1 1

MmpL 3

CmpL1

MmpL13a

\section{MmpL1}

MmpL2

MmpL 9

MmpL 5

MmpL 4

MmpL12

MmpL10

MmpL 8

MmpL11

MmpL3

CmpL1

MmpL13a
TMS-2

TMS-3

$\begin{array}{ll}\text { TGISIAVIA IMLFIAYRSLSAALIMLLTVGLELLAVRGIISTFAVNDLMGLSTFTVNVLV } & 256 \\ \text { TAITSMVIAAMLLVIYRSVITAVLVLIMVGIDLGAIRGFIALLADHNIFSLSTFATNLLV } & 257 \\ \text { TGLTFAVITVLLLLVYRSIATTLLILPMVFIGLGATRGTIAFLGYHGMVGLSTFVVNILT } & 262 \\ \text { EAVTFTVI IVMLLLVYRSI ITSAIMLTMVVLGLLATRGGVAFLGFHRIIGLSTFATNLLV } & 267 \\ \text { TMVTVAVIFIMLLLVYRSI ITVVLLLITVGVELTAARGVVAVLGHSGAIGLTTFAVSLLT } & 263 \\ \text { EIGTAVSVLIILILVYRNLVTMLVPLATIGASVVTAQGTLSGL-AEFGLAVNMQAIVFMS } & 259 \\ \text { ELAIAVMLLVILMVIYRNPVTMLLPLVTIGASLMTAQALVAGVSLVGGLAVSNQAIVLLS } & 233 \\ \text { EFAITILLLVILLIIYGNPITMVLPLITIGMSVVVAQRLVAIA-GLAGLGIANQSIIFMS } & 277 \\ \text { ERWNLPIILMVLVAVFGSLAAAAIPLALAVCTVVITMGLVFVLSMHT--TMSVFVTSTVS } & 239 \\ \text { EVLALPLVAVVLFFVFGGVIAAGLPVMVGGLCIAGALGIMRFLAIFG--PVHYFAQPVVS } & 242 \\ \text { EVFALPFVAILLLIVFGSVVAAAMPLIVGILSILGSLGILAILAGFF--QVNVFAQSVVT } & 244 \\ \text { ELIAIPLSFLVIIWVFGGLLAAGLPMAQAVLAVVGSMAVLRLVTFAT--EVSTFALNLST } & 239\end{array}$

TMS-4

TMS- 5
316

317

322

327

323

319

293

337

299

302

304

299

TMS- 6

$\begin{array}{ll}\text { RLPYFNTLASPCAIGLVTVMLASLTLAPAIIAVASRFG } & 354 \\ \text { RLPYFETLGAPIAIGMLVAVLAALTLGPAVLTVGSFF } & 355 \\ \text { RLTLFHTMGPPLAIGMLVSVAAALTLAPAIAIAGRFG } & 360 \\ \text { RLPYFQTLGVPLAIGMVIVVAAALTLGPAIAVTSRFG } & 365 \\ \text { RMPYFQTLGIPCAVGMLVAVAVALTLGPAVLHVGSRF } & 361 \\ \text { KLEVFSAVGPAIAVAITVSLLGAVTLLPAILTLTGRRG } & 357 \\ \text { KLGVFSTVGPALAIGIAVSFLAAVTLLPAILVLASPRG } & 331 \\ \text { QLGILKTVGPMLGISVAVVFFAATLLPALMVLTGRRG } & 375 \\ \text { NTPALRSMATGAILAVAVAMLTSATLTPAVLATFARAA } & 337 \\ \text { PQGFLKSLTYATIASVMLSAILSITVLPACLGILGKHV } & 340 \\ \text { PQAFLKSVAFGAISAVGLAALMSVTVLPSLFSMLGKNI } & 342 \\ \text { RCTF------------------------------- } & 303\end{array}$


(b)

MmpL1

MmpL 6

MmpL2

MmpL 9

MmpL 5

MmpL 4

MmpL12

MmpL10

MmpL 8

MmpL11

MmpL 3

CmpL1

MmpL13B

MmpL 6

MmpL 9

MmpL5

MmpL 4

MmpL12

MmpL10

MmpL 8

MmpL11

MmpL3

CmpL1

MmpL13B loop 2

GTPLQAAS IYLAGTSSTYKDI
GTPMAGAGIYLAGTAATFKDI
GTPLQGAAIYLGGTAATFKDI
ATPLQGAKIYIGGTAATYQDIR
GTPLEGSAIYLGGTAAMFKDI
GTPLEDAKIYLAGTAAVFHDI
NTELEDAT IGLAGVPTALRDI
NTSLADAS ISMSGYPVMLRDI
NTALADAKVSVVGLPVVLKDTR
VAG--AAQVDVGGPTALIKDE
ITPPKGITVLVGGTPALELDS
ISVPEGVEVQIGGTPAMEIES I
VAGPAGRSVVMAGVAQVNRDS

TMS- 9
TMS- 8

$\overline{\text { HEGTLYDVMIAVVASLCLIFI IMLGITRSVVASAVIVGT }}$ IQDGATYDLLIAGIAALSLILLIMMI ITRSLVAALVIVGT IREGARYDLLIAGVAA ISLILI IMMI ITRSVVAAVVIVGT IRDGTKYDILIVGIAAVCLVFIVMLMITQSLIASLVIVGT LSDGNTYDLMIAGISALCLIF I IMLITTRSVVAAAVIVGT ISEGAQWDLLIAA ISSLCLIFI IMLI ITRAF IAAAVIVGT IRDYYNSDMKF IVIATIVIVFLILVILLRALVAPIYLIGS IRDYYERDMRLIVAVTVVVVI L ILMALLRA IVAPLYLVGS TRDYS DHDLRLI IAMTVCIVLLILIVLLRA IVAPLYLIGS FDDRVSATQPLVLVFVAVIAFLMLLISIRSVFLAFKGVLM S I HGLFAKMPLMVV I LLTTT IVLMFLAFGSVVLP IKATLM S IEALFEKLLWMALY IVLATF I LMALVFGSVILPAKA I IM SVDAVTDRLPMVLGLIAAITYVLLFLLTGSVVLPAKALVC
795

223

796

801

807

802

855

834

902

559

590

550

263
MmpL2

\begin{tabular}{l}
\hline VALSLGSAFGLSVLIWQHIL-------HMPLHWLVLPMAI IVMLAVGSDYNLLLIARFQ \\
VALSLGASFGLSVLVWQHLL-------GIQLYWIVLALAVI LLLAVGSDYNLLLISREK \\
VVLSMGASFGLSVLVWQDIL-------GIELYWMVLAMSVI LLLAVGSDYNLLLISRLK \\
VLLSLGTAFGLSVLIWQHFV-------GLQVHWT IVAMSVIVLLAVGSDYNLLLVSRFK \\
VVLSLGASFGLSVLIWQHIL-------GIELHWLVLAMAVI ILLAVGADYNLLLVARLK \\
VALSLGASFGLSVLLWQHIL-------AIHLHWLVLAMSVIVLLAVGSDYNLLLVSRFK \\
VLISYLSALGIGTLVFQLIL-------GQEMHWSLPGLSFILLVAIGADYNMLLISRIR \\
VVISYMSAIGLGVVVFQVFL-------GQELHWSVPGLAFVVLVAVGADYNMLLASRLR \\
VIVSYLAALGIGVIVFQFLL-------GQEMHWS IPGLTFVILVAVGADYNMLLISRLR \\
TLLSVAAAYGSLVMVFQWGWARGLGFPALHSIDSTVPPLVLAMTFGLSMDYEIFLLTRIR \\
SALTLGSTMGILTWIFVDGHFSKWLNFTPTPLTAPVIGLI IALVFGLSTDYEVFLVSRMV \\
TILGMGATLGILTLMFVDGVGASALNFSPGPLMSPVLVLIMAIIYGLSTDYEVFLVSRMV \\
NVLSLTAAFGALVWIFQEGHFGALGTTPSGTLVANMPVLLFCIAFGLSMDYEVFLVSRIR
\end{tabular}

TMS-11

MmpL 1

MmpL 6

MmpL2

MmpL 9

MmpL 5

MmpL 4

MmpL12

MmpL1 0

MmpL 8

MmpL11

MmpL3

CmpL1

MmpL13B

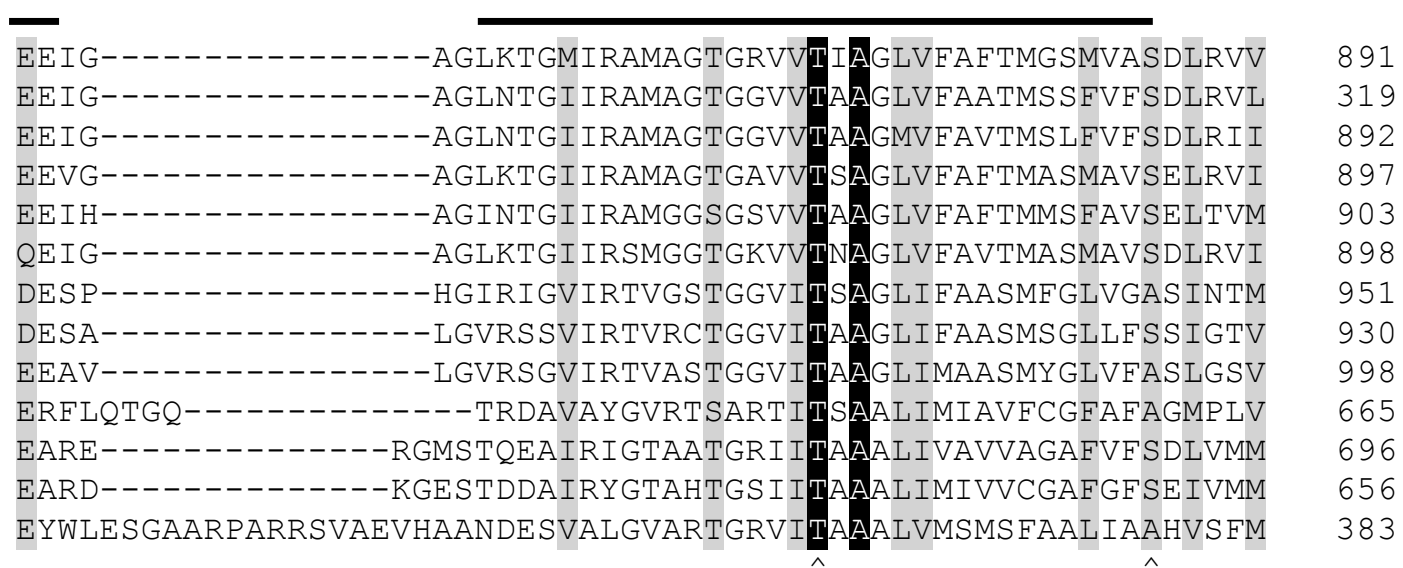


MmpL1

MmpL 6

MmpL2

MmpL 9

MmpL5

MmpL 4

MmpL12

MmpL1 0

MmpL 8

MmpL11

MmpL3

CmpL1

MmpL13B

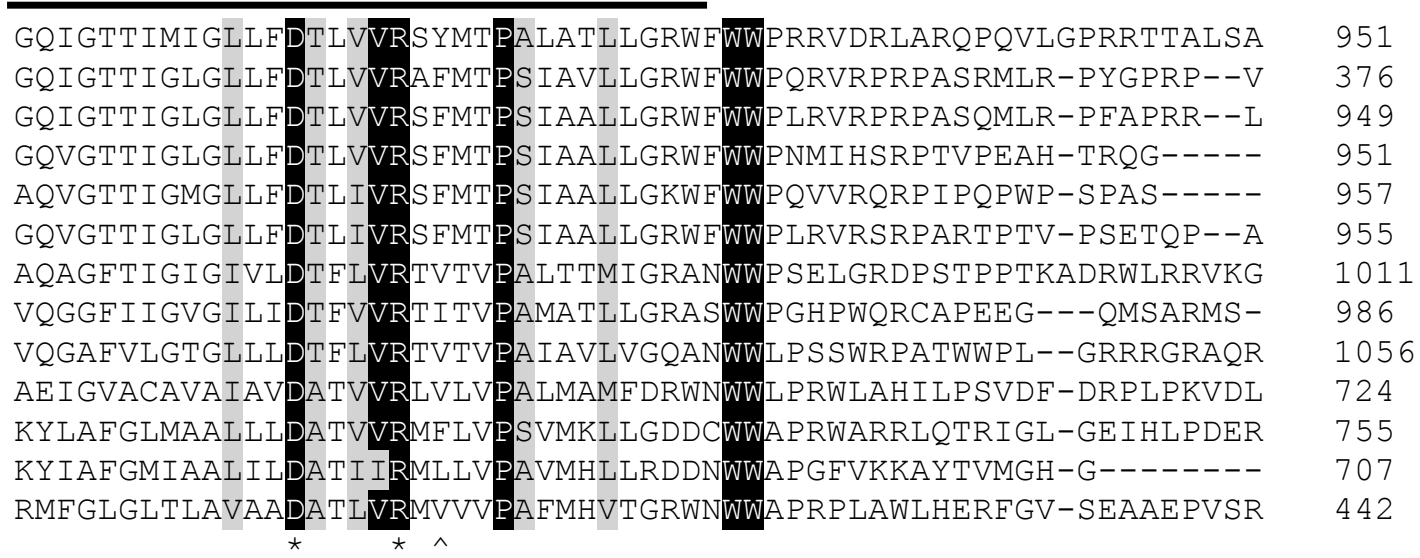


Figure S5: Effect of thiol-reactive probes on mycolic acid export by Msmg $\Delta m m p L 3$ strains expressing MmpL3 mutants with cysteine substitutions at positions Q40, Y235, P625 and V694.

(a) Structures of MTSES and ebselen.

(b) TMM and TDM synthesis in untreated and ebselen $(10 \mu \mathrm{g} / \mathrm{ml})$ - or MTSES $(512 \mu \mathrm{g} / \mathrm{ml})$-treated Msmg $\Delta m m p L 3$ expressing Q40C, Y235C, P625C and V694C $\Delta$ Cys-MmpL3 variants was determined by metabolic labeling with [1,2-

$\left.{ }^{14} \mathrm{C}\right]$ acetic acid. Radiolabeled lipids from treated and untreated cells were analyzed by TLC and the amount of TMM and TDM in each sample semi-quantified using a PhosphorImager. Results are representative of one typical experiment and are expressed as fold-increases in the ratio of TMM to TDM in each treated cells relative to untreated cells.

The different accessibility of residues to MTSES and ebselen is likely to account for the only partial overlap between the residues revealed by the two structurally different probes.

$\mathbf{a}$<smiles>O=c1c2ccccc2[se]n1-c1ccccc1</smiles>

MTSES<smiles>CS(=O)(=O)SCCS(=O)(=O)O[NH3+]</smiles>

b

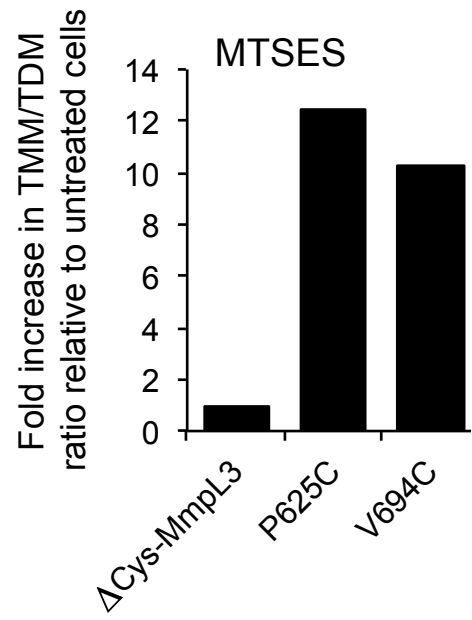

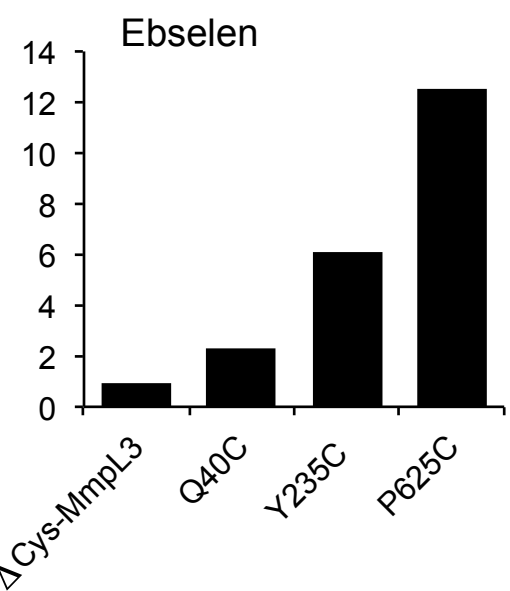




\section{Table S1: I-TASSER free modeling.}

Top structural analogs close to the CmpL1 target in the PDB and considered having the same topological fold whenever TM-score $>0.5$ (Xu and Zhang, 2010).

\begin{tabular}{|c|c|c|c|c|c|c|}
\hline $\operatorname{Rank}^{\mathrm{a}}$ & $\begin{array}{c}\text { PDB } \\
\text { Hit }\end{array}$ & Protein & $\begin{array}{c}\text { TM- } \\
\text { score }\end{array}$ & $\mathrm{RMSD}^{\mathrm{b}}$ & $\mathrm{IDEN}^{\mathrm{c}}$ & $\operatorname{Cov}^{d}$ \\
\hline 1 & 3aqp & $\begin{array}{c}\text { SecDF, T. thermophilus } \\
\text { translocon-associated } \\
\text { membrane protein }\end{array}$ & 0.737 & 1.40 & 0.112 & 0.749 \\
\hline 2 & $3 d 9 b$ & $\begin{array}{l}\text { AcrB, E. coli } \text { Multidrug } \\
\text { Efflux transporter }\end{array}$ & 0.578 & 5.29 & 0.097 & 0.693 \\
\hline 3 & $4 \mathrm{mt} 1$ & $\begin{array}{l}\text { MtrD, N. gonorrhea, } \\
\text { Multidrug Efflux Pump }\end{array}$ & 0.575 & 5.27 & 0.096 & 0.692 \\
\hline 4 & 3 ne 5 & $\begin{array}{c}\text { CusB } \boldsymbol{A}, \text { E. coli heavy-metal } \\
\text { Efflux complex }\end{array}$ & 0.565 & 5.11 & 0.101 & 0.672 \\
\hline 5 & $4 \mathrm{k} 0 \mathrm{e}$ & $\begin{array}{l}\text { ZneA, C. metallidurans } \\
\mathrm{Zn}(\mathrm{II}) / \text { proton antiporter }\end{array}$ & 0.553 & 5.19 & 0.099 & 0.661 \\
\hline
\end{tabular}

${ }^{a}$ Ranking of proteins is based on TM-score of the structural alignment between the query structure and known structures in the PDB library (Zhang and Skolnick, 2004).

${ }^{\mathrm{b}}$ RMSD is the RMSD between residues that are structurally aligned by TM-align. TM-align is an algorithm for sequence-order independent protein structure comparisons (Zhang and Skolnick, 2005).

${ }^{\mathrm{c}}$ IDEN is the percentage sequence identity in the structurally aligned region.

${ }^{\mathrm{d}} \mathrm{Cov}$ represents the coverage of the alignment by TM-align and is equal to the number of structurally aligned residues divided by length of the query protein. 
Table S2: Drug susceptibility of Msmg $\Delta m m p L 3$ strains expressing mutated variants of $\Delta$ CysMmpL3.

MICs (in $\mu \mathrm{g} / \mathrm{ml}$ ) were determined in 7H9-ADC broth by using the resazurin blue test and visually scanning for growth. MRP, meropenem; AMP, ampicillin; INH, isoniazid; CIP, ciprofloxacin; RIF, rifampicin.

Four- (or greater) fold increases in susceptibility are highlighted in orange.

\begin{tabular}{lccccc}
\hline Strain & MRP & AMP & INH & CIP & RIF \\
\hline$\Delta$ Cys-MmpL3 (parent strain) & 4 & 256 & $4-8$ & 0.25 & 16 \\
G253C & 2 & 128 & 4 & 0.25 & 4 \\
R259C & 2 & 128 & 8 & 0.25 & 8 \\
S288T & 0.5 & 64 & 8 & 0.25 & 4 \\
Q304C & 2 & 128 & 8 & 0.25 & 8 \\
D550C & 1 & 64 & 4 & 0.25 & 4 \\
P625C & 2 & 128 & 4 & 0.25 & 8 \\
T675C & 2 & 256 & 8 & 0.25 & 8 \\
S691C & 1 & 128 & 8 & 0.25 & 4 \\
\hline
\end{tabular}

Movie S1: Movie showing the 3D EM negative staining map and the superposition of the CmpL1 structural model refined against the EM map.

In the last part of the movie, a detailed fit is provided of the model to the EM map by moving the front clipping plane of the image down and up the 3-fold axis. Surface of the EM map shown was contoured at relative level of 3. 


\section{Supplementary Methods}

Bacterial strains and growth conditions - M. smegmatis (Msmg) $\mathrm{mc}^{2} 155$ was grown in Middlebrook $7 \mathrm{H} 9$ broth (Difco) with $10 \%$ albumin-dextrose-catalase (ADC) supplement and $0.05 \%$ Tween 80 and on $7 \mathrm{H} 11$-ADC agar at 30 or $37^{\circ} \mathrm{C}$. Where required, kanamycin (Kan) $(25 \mu \mathrm{g} / \mathrm{ml})$, hygromycin $(\mathrm{Hyg})(50 \mu \mathrm{g} / \mathrm{ml})$, sucrose $(10 \%)$ and anhydro-tetracycline (ATc) $(0.1$ to $50 \mathrm{ng} / \mathrm{ml})$ were added to the culture medium. Cgl ATCC 13032 and LY $108{ }^{4}$ were grown at $30^{\circ} \mathrm{C}$ in brainheart infusion (BHI) medium supplemented with $2 \%(\mathrm{w} / \mathrm{v})$ glucose or $9.1 \%(\mathrm{w} / \mathrm{v})$ sorbitol, or in the minimal medium (20.9 $\mathrm{g}$ of MOPS, $5 \mathrm{~g}$ of $\left(\mathrm{NH}_{4}\right)_{2} \mathrm{SO}_{4}, 5 \mathrm{~g}$ of urea, $1 \mathrm{~g}$ of citrate, $0.5 \mathrm{~g}$ of $\mathrm{K}_{2} \mathrm{HPO}_{4}, 0.5 \mathrm{~g}$ of $\mathrm{KH}_{2} \mathrm{PO}_{4}, 0.25 \mathrm{~g}$ of $\mathrm{MgSO}_{4} \cdot 7 \mathrm{H}_{2} \mathrm{O}, 10 \mathrm{mg}$ of $\mathrm{CaCl}_{2}, 10 \mathrm{mg}$ of $\mathrm{MnSO}_{4} \cdot \mathrm{H}_{2} \mathrm{O}, 10 \mathrm{mg}$ of $\mathrm{FeSO}_{4} \cdot 7 \mathrm{H}_{2} \mathrm{O}, 10 \mathrm{mg}$ of thiamine, $1 \mathrm{mg}$ of $\mathrm{ZnSO}_{4} \cdot 7 \mathrm{H}_{2} \mathrm{O}$, $0.2 \mathrm{mg}$ of $\mathrm{CuSO}_{4}, 0.2 \mathrm{mg}$ of biotin per liter, $\mathrm{pH} \mathrm{7.3)} \mathrm{with} 1 \%$ glucose. E. coli $\mathrm{DH} 5 \alpha$ and $\mathrm{S} 17-1$ were grown at $37^{\circ} \mathrm{C}$ in Luria Bertani (LB) medium (10 g/l Bacto-tryptone, $5 \mathrm{~g} / \mathrm{l}$ yeast extract and $5 \mathrm{~g} / \mathrm{l} \mathrm{NaCl})$. Kan $(25 \mu \mathrm{g} / \mathrm{ml})$ or tetracycline $(10$ $\mu \mathrm{g} / \mathrm{ml}$ for DH5 $\alpha$ and $20 \mu \mathrm{g} / \mathrm{ml}$ for $C g l)$ were added as needed.

Expression and purification of MmpL3 and CmpL1 in Cgl-Cgl cmpL1 and Mtb mmpL3 were expressed in Cgl ATCC 13032 from the multicopy expression plasmid pAN6. ${ }^{50}$ Freshly transformed $C g l$ cells were inoculated into $100 \mathrm{~mL}$ of $2 \times \mathrm{TY}$ medium (16 g of Bacto-tryptone, $10 \mathrm{~g}$ of yeast extract and $5 \mathrm{~g}$ of $\mathrm{NaCl}$ per liter) supplemented with $2 \%(\mathrm{w} / \mathrm{v})$ glucose and $25 \mu \mathrm{g} / \mathrm{ml} \mathrm{Kan}$ and incubated at $30^{\circ} \mathrm{C}$ until $\mathrm{OD}_{600}$ reached $\sim 0.5$. Protein expression was induced by $0.1 \mathrm{mM}$ IPTG for 8 hours. Cells were collected by centrifugation at $4,000 \mathrm{x} g$ at $4^{\circ} \mathrm{C}$ for 20 min and resuspended in $50 \mathrm{ml}$ buffer containing $10 \mathrm{mM}$ Tris- $\mathrm{HCl}, 1 \mathrm{mM} \mathrm{MgCl}, 1 \mathrm{mM}$ phenylmethylsulfonyl fluoride (PMSF), protease inhibitor tablets (Sigmafast $^{\mathrm{TM}}$ ), and $100 \mu \mathrm{g} / \mathrm{ml}$ DNase I ( $\mathrm{pH}$ 8.0). Cells were broken using a French Press FA078 apparatus (SIM Instruments. Inc.). Unbroken cells were removed by low-speed centrifugation at $4,000 \mathrm{x} g$ at $4^{\circ} \mathrm{C}$ for 10 min. Total membrane fractions were isolated by ultracentrifugation at $40,000 \mathrm{x}$ for $1 \mathrm{~h}$ at $4^{\circ} \mathrm{C}$. Membrane proteins were solubilized in $10 \mathrm{ml}$ of $100 \mathrm{mM}$ Tris- $\mathrm{HCl}(\mathrm{pH} 8.0), 150 \mathrm{mM} \mathrm{NaCl}, 1 \mathrm{mM}$ EDTA, $1 \mathrm{mM}$ PMSF, protease inhibitor tablets (Sigmafast $^{\mathrm{TM}}$ ), and $1 \% \mathrm{n}$-dodecyl $\beta$-D-malto-pyranoside (DDM) for $3 \mathrm{~h}$ on ice. After removal of the insoluble fraction by ultracentrifugation, solubilized proteins were loaded onto a 0.4-ml column packed with Strep-tactin Superflow Agarose resin (IBA, Solutions for Life Sciences). CmpL1 was purified from soluble fractions using the manufacturer's protocol modified to include $0.03 \%$ DDM in all buffers. Collected fractions were pooled and concentrated by an Amicon concentrator (Membrane NMWL, $100 \mathrm{kDa}$ ). The concentrated fraction was subsequently ultracentrifuged (Sorvall Discovery M150 SE) at 200,000 $\mathrm{xg}$ for $1 \mathrm{~h}$ at $4^{\circ} \mathrm{C}$. The authenticity of CmpL1 was confirmed by cutting-out the band corresponding to the purified fraction of CmpL1 of the SDS-PAGE gel, followed by in-gel digestion with trypsin, and mass spectrometry analysis. Comparison of the resulting peptide sequences predicted from mass spectrometry analysis with those of $\mathrm{CmpL1}$ uniquely identified the purified fraction as the CmpL1 transporter. For gel filtration chromatography, protein samples were loaded onto a size exclusion column (Superdex 200 GE 3.2/300) equilibrated in

eluting buffer (100 mM Tris $\mathrm{HCl}$ (pH 8.0), $150 \mathrm{mM} \mathrm{NaCl}, 1 \mathrm{mM}$ PMSF, 0.03\% (w/w) DDM) using a GE micro ÄKTA system. Protein concentrations were determined from $\mathrm{OD}_{280}$ measurements using a GE NanoPhotometer ${ }^{\mathrm{TM}}$ Pearl spectrophotometer. At this wavelength, DDM has negligible absorbance. The final $\mathrm{CmpL} 1$ protein yield is approximately $2 \mathrm{mg}$ for a 25 liter $\mathrm{Cgl}$ culture. The fraction corresponding to $\mathrm{CmpLl}$ after size exclusion chromatography was then used for EM negative staining.

For chemical cross-linking, purified $\mathrm{CmpL1}$ or MmpL3 proteins were treated with increasing concentrations of dithiobis (succinimidylpropionate) (DSP, Pierce) (Krishnamoorthy et al., 2013). Crosslinking reactions were carried out at $37{ }^{\circ} \mathrm{C}$ for $30 \mathrm{~min}$ and terminated by addition of Tris- $\mathrm{HCl}(\mathrm{pH} 7.5)$ to a final concentration of $20 \mathrm{mM}$.

Site-directed mutagenesis and generation of C-terminal truncated mutants of MmpL3 - $\Delta$ Cys-MmpL3, in which Cys24, Cys332 and Cys 730 of MmpL3 were replaced by serine residues, was generated by the two-step PCR overlap method and pAN6-mmpL3 as the DNA template. The cysteine-mutated variants of $\triangle C y s-m m p L 3$ were next generated by the same method using pMVGH1- $\triangle C y s-m m p L 3$ as the DNA template and then cloned into the NdeI and NheI restriction sites of pAN6. Some of the mutants were obtained from GenScript (Piscataway, NJ, USA). Unique Cys residues were introduced at the following positions of $\Delta$ Cys-MmpL3: 9, 33, 40, 103, 105, 124, 146, 188, 205, 208, 225, 235, 249, 251, 252, 253, $255,259,267,273,288,304,309,320,327,330,331,350,365,382,402,413,467,507,543,550,567,587,600,615$, $625,640,641,645,648,675,677,684,691,694,700,710,714,715,717,720,731,732$, and 845 . 
The C-terminal truncated forms of MmpL3 were obtained by PCR amplification of the mmpL3 gene from Mtb H37Rv using as the upstream primer an oligonucleotide matching the 5'-end of $m m p L 3$ and as downstream primers oligonucleotides that introduced stop codons as positions 721, 744, 750, 762, 777, 859 and 915 of the protein. PCR fragments were cloned in the mycobacterial expression plasmid, pMVGH1. ${ }^{27}$

Transmembrane topology mapping of MmpL3 - Cgl LY108 cells harboring pAN6 expression plasmids were grown in 150 $\mathrm{mL}$ of $2 \times \mathrm{TY}$ medium supplemented with $2 \%(\mathrm{w} / \mathrm{v})$ glucose and, depending on the plasmid, with $25 \mu \mathrm{g} / \mathrm{ml} \mathrm{kanamycin}$ or $20 \mu \mathrm{g} / \mathrm{ml}$ tetracycline. Cells were incubated at $30^{\circ} \mathrm{C}$ with shaking until $\mathrm{OD}_{600}$ reached $\sim 0.5$. Protein production was induced with $0.1 \mathrm{mM}$ IPTG and cells were grown until $\mathrm{OD}_{600}$ reached $\sim 3$. Membrane fractions were separated as described above and treated with $0.5 \mathrm{mM}$ Fluorescein-5-Maleimide (FM) in PBS buffer (pH 7.5) followed by incubation for $60 \mathrm{~min}$ at $4^{\circ} \mathrm{C}$. The reaction was terminated by addition of $10 \mathrm{mM}$ DTT and incubation for $5 \mathrm{~min}$. The membrane fraction was recovered by ultracentrifugation at $40,000 \times \mathrm{g}$ for $1 \mathrm{~h}$ at $4^{\circ} \mathrm{C}$ and $\mathrm{MmpL} 3$ Cys-mutated variants were purified by anti-Strep affinity chromatography as described above prior to separation on 10\% SDS-PAGE gel. The fluorescence was measured using Storm ${ }^{\mathrm{TM}}$ (GE Healthcare) and quantified using the ImageJ program (NIH). Protein expression was analyzed by silver nitrate staining.

To further determine the subcellular localization of the soluble loops and in C-terminal end of MmpL3 (Msmg ortholog, MSMEG_0250) expressed in Msmg, three C-terminal MmpL3-GFP fusions, one fusing MmpL3 to GFP at residue 171 (in loop 1), one at residue 563 (in loop 2), and one at the C-terminal end of MmpL3 (residue 1013), were generated in the expression plasmids $\mathrm{pJB}(-)$ and $\mathrm{JB}(+){ }^{14}$ and expressed from the phsp60 promoter in Msmg. In $\mathrm{pJB}(+)$, a single TMS from glycophorin $\mathrm{A}$ is added between the C-terminal fusion point of the protein of interest and the GFP to convert membrane proteins with extracellular C-termini to proteins with intracellular C-termini. Because GFP fluoresces in the cytoplasm but not in the periplasm, a high fluorescence signal in the $\mathrm{pJB}(-)$ version and background fluorescence in the $\mathrm{pJB}(+)$ version are indicative of the fusion point being cytoplasmic. Opposite fluorescence intensities indicate, on the contrary, that the fusion point is localized in the periplasm. Cultures of transformants grown to $\log$ phase $\left(\mathrm{OD}_{600} \approx 0.8\right)$, washed twice with PBS and resuspended in $100 \mu \mathrm{l}$ of the same buffer were transferred to black 96-well plates with transparent bottoms (Corning) and their fluorescence determined using a 2030 MultiLabel Reader Victor X5 plate reader (Perkin Elmer) at excitation and emission wavelengths of $485 \mathrm{~nm}$ and $535 \mathrm{~nm}$, respectively. The fluorescence value of each sample was normalized to the $\mathrm{OD}_{600}$ of the culture.

Negative staining EM - CmpL1 samples (each $5 \mu \mathrm{L}$ diluted to $50 \mu \mathrm{g} / \mathrm{mL}$ ) were applied onto negatively glow-discharged carbon-coated grids (400 mesh, copper grid) for 1 minute and excess liquid was removed by blotting with filter paper. Freshly prepared $1.5 \%$ uranylformate $(\mathrm{pH} 5)$ was added $(5 \mu \mathrm{l})$ for 1 minute and then blotted. Digital micrographs were collected using a FEI Tecnai G2 F20 microscope operated at $200 \mathrm{kV}$ and equipped with a Gatan Ultrascan 4k x 4k Digital CCD Camera. The images were recorded at defocus between $0.7-1.4 \mu \mathrm{m}$ at a magnification of $134,010 \mathrm{X}$ at the camera and a pixel size of $1.12 \AA$.

Contrast transfer function parameters were determined using CTFFIND4 (Rohou et al., 2015). Protein particles were boxed an in-house software using a template. The template was a disc obtained by averaging $\sim 100$ particles oriented randomly. After extraction, 21,033 particles were binned $2 \mathrm{X}$ for a final box size of 128x128 pixels and a resulting pixel size of $2.24 \AA$ at specimen level. False positives $(\sim 2,000$ particles $)$ were first removed from the particle stack after a $2 \mathrm{D}$ classification $(\mathrm{K}=16)$ with RELION-1.3 software suite (Scheres et al., 2012). The reference 3D map was obtained using Simple Prime (Elmlund et al., 2013) with the clean particles. RELION-1.3 software suite (Scheres et al., 2012) was used for the 3D analysis. The CTF was applied by phase flipping only (no amplitude correction). The first step consisted of a maximum likelihood 3D classification with 3 seeds and a tau of 2. Class $1(6,701$ particles) and class $3(5,251$ particles $)$ were similar, whereas class 2 (6,288 particles) was poorly defined with accumulation of densities on the symmetry axis. Classes 1 and 3 were refined further using the Relion autorefine routine while applying the C3 symmetry. The routine stopped at the angular sampling of 3.5 degrees. The resulting density features were highly similar for classes 1 and 3 , and class 1 was chosen for subsequent structural modeling. 3D-EM density maps were visualized using Chimera (Pettersen et al., 2004). The resolution of the map was estimated to be $18 \AA$ using the gold standard FSC criterion of 0.5.

Structural modeling - I-TASSER was used to prepare a model for the 3D structure of CmpL1 based on its primary amino acid sequence. ${ }^{51}$ Although $a b$ initio structural modeling is a challenge for folding of proteins with $>250$ amino acids 
(Zhang, 2014), TM helices due to their hydrophobicity and their length of about 20 amino acids are easier to predict and simplify ab initio modeling by reducing the conformational search space. The structure homology predictions for CmpL 1 by I-TASSER are shown in Table 1. Even though the CmpL1 sequence homology with the target structures are low $(<$ $12 \%$ identity), the top ranked target, SecDF, a Thermus thermophilus protein secretion-associated membrane protein, ${ }^{36}$ was demarcated by its high TM-score of 0.74 and low RMSD value of 1.40 , in comparison to the various efflux pumps which had lower TM-scores and significantly greater RMSD values. The trimeric quaternary structure of CmpL1 was next built using the second best scoring structure, AcrB, (PDB ID: 3D9B) as SecDF is a monomeric transmembrane protein. The few subunit clashes were relieved by manual adjustments in PyMol (The PyMOL Molecular Graphics System, Version 1.8 Schrödinger, LLC) and, by using the program FG-MD (a molecular dynamics (MD)-based algorithm for atomic-level protein structure refinement), the local geometry was improved by relieving steric clashes and refining torsion angles and hydrogen-binding networks (Zhang et al., 2011). Additional geometry regularization used the geometry minimization model tool in Phenix (Adams et al., 2010) and Coot (Emsley et al., 2010).

The refined structure of CmpL1 was then used for template-based modeling of the MmpL3 sequence by I-TASSER yielding an initial structural model for the MmpL3 protomer and, consequently, of its trimeric quaternary structure. $A b$ initio modeling by I-TASSER of the MmpL3 protomer predicted structural homology with SecDF. However, using this structural model for the MmpL3 subunit to build the trimeric structure resulted in unacceptable steric clashes among the interacting periplasmic N-terminal domain PN (residues 34-174) of each subunit in this MmpL3 trimer. The templatebased trimeric structural model for MmpL3 was then relaxed using FG-MD followed by geometry regularization in Phenix and with Coot to obtain the final quaternary structure.

MmpL3 activity assay in intact Msmg cells - The activity of the different point-mutated or truncated MmpL3 mutants was determined by testing their ability to rescue the growth of a Msmg $\Delta m m p L 3$ knock-out mutant. To this end, the pMVGH1 constructs expressing the different $m m p L 3$ mutants were used to transform a Msmg strain having undergone a single crossover event at its $m m p L 3$ locus, ${ }^{27}$ and the resulting transformants were next plated on $7 \mathrm{H} 11$-ADC agar containing Kan, Hyg and sucrose to select for allelic exchange mutants. Failure to isolate allelic exchange mutants at 30 and $37^{\circ} \mathrm{C}$ is indicative of the lack of activity of the mmpL3 mutant expressed from pMVGH1.

The susceptibility of Msmg $\Delta m m p L 3$ knock-out mutants expressing Cys-mutated variants of $m m p L 3$ (52 strains in total) to the thiol-reactive probes, ebselen (Sigma) and 2-sulfonatoethyl methanethiosulfonate (MTSES) (Cayman Chemicals) was determined in 96 -well microtiter plates in $7 \mathrm{H} 9$-ADC broth at $37^{\circ} \mathrm{C}$ by visually scanning for growth.

Construction of a tmaT knock-down mutant of Msmg - A conditional knock-down mutant of Msmg expressing MSMEG_0319 (tmaT) under control of an anhydro-tetracycline-inducible promoter was constructed by homologous recombination. A two-step procedure employing the temperature-sensitive plasmid pPR27-XylE expressing the counterselectable marker $s a c B$ was used to achieve allelic replacement at the tmaT locus of Msmg $\mathrm{mc}^{2} 155$ (Jackson et al., 2001). pSETetR-tmaT, an episomal rescue plasmid in which the tmaT gene from Msmg was placed under control of an ATc-inducible promoter, was constructed by inserting the entire coding sequence of tmaT into pSETetR. ${ }^{27}$ Primer sequences for the knock-down construct are available upon request.

Whole cell radiolabeling experiments - Radiolabeling of whole $M s m g$ cells $(0.5 \mu \mathrm{Ci} / \mathrm{ml}$; specific activity, $54.3 \mathrm{Ci} / \mathrm{mol}$, Perkin Elmer) was performed in $7 \mathrm{H} 9$-ADC-Tween 80 medium for $3 \mathrm{hr}$ at $37^{\circ} \mathrm{C}$ with shaking. $\left[1,2-{ }^{14} \mathrm{C}\right]$ acetic acid was added at the same time as the thiol-reactive probes (ebselen or MTSES). For labeling of the tmaT conditional knockdown, washed cell pellets were resuspended to an $\mathrm{OD}_{600}$ of 0.1 in $7 \mathrm{H} 9-\mathrm{ADC}-\mathrm{Tween} 80$ medium containing 0 to $10 \mathrm{ng} / \mathrm{ml}$ ATc and the cells labeled with $\left[1,2-{ }^{14} \mathrm{C}\right]$ acetic acid for $3 \mathrm{hr}$ at $37^{\circ} \mathrm{C}$.

Analytical procedures - Total lipids extraction from bacterial cells and preparation of fatty acid and mycolic acid methyl esters from extractable lipids and delipidated cells followed earlier procedures. ${ }^{27}\left[1,2-{ }^{14} \mathrm{C}\right]$ acetic acid-derived lipids and fatty acid/mycolic acid methyl esters were separated by TLC on aluminum-backed silica gel 60-precoated plates $\mathrm{F}_{254}(\mathrm{E}$. Merck) and revealed by PhosphorImaging. 


\section{Supplementary References}

Adams, P.D., Afonine, P.V., Bunkoczi, G., Chen, V.B., Davis, I.W., Echols, N., Headd, J.J., Hung, L.W., Kapral, G.J., Grosse-Kunstleve, R.W., et al. (2010). PHENIX: a comprehensive Python-based system for macromolecular structure solution. Acta Crystallogr. D. Biol. Crystallogr. 66, 213-221.

Elmlund, H., Elmlund, D., and Bengio, S. (2013). PRIME: probabilistic initial 3D model generation for single-particle cryo-electron microscopy. Structure 21, 1299-1306.

Emsley, P., Lohkamp, B., Scott, W.G., and Cowtan, K. (2010). Features and development of Coot. Acta Crystallogr. D. Biol. Crystallogr. 66, 486-501.

Jackson, M., Camacho, L.R., Gicquel, B., and Guilhot, C. (2001). Gene Replacement and Transposon Delivery Using the Negative Selection Marker $s a c B$. In Mycobacterium tuberculosis protocols, T. Parish \& N.G. Stocker, Eds (Humana Press, Totowa N J), 54, pp. 59-75.

Krishnamoorthy, G., Tikhonova, E. B., Dhamdhere, G., and Zgurskaya, H. I. (2013) On the role of TolC in multidrug efflux: the function and assembly of AcrAB-TolC tolerate significant depletion of intracellular TolC protein. Mol. Microbiol. 87, 982-997.

Pettersen, E.F., Goddard, T.D., Huang, C.C., Couch, G.S., Greenblatt, D.M., Meng, E.C., and Ferrin, T.E. (2004). UCSF Chimera-a visualization system for exploratory research and analysis. J. Comput. Chem. 25, 1605-1612.

Rohou, A., and Grigorieff, N. (2015). CTFFIND4: Fast and accurate defocus estimation from electron micrographs. J. Struct. Biol. 192, 216-221.

Scheres (2012). RELION: implementation of a Bayesian approach to cryo-EM structure determination. J. Struct. Biol. 180, 519-530.

$\mathrm{Xu}$, J., and Zhang, Y. (2010). How significant is a protein structure similarity with TM-score $=0.5$ ? Bioinformatics 26 , 889-895.

Zhang, Y. (2014). Interplay of I-TASSER and QUARK for template-based and ab initio protein structure prediction in CASP10. Proteins 82 Suppl 2, 175-187.

Zhang, Y., and Skolnick, J. (2004). Scoring function for automated assessment of protein structure template quality. Proteins 57, 702-710.

Zhang, Y., and Skolnick, J. (2005). TM-align: a protein structure alignment algorithm based on the TM-score. Nucleic Acids Res. 33, 2302-2309.

Zhang, J., Liang, Y., and Zhang, Y. (2011). Atomic-level protein structure refinement using fragment-guided molecular dynamics conformation sampling. Structure 19, 1784-1795. 